Gefässchirurgie 2014 · 19:611-613

DOI 10.1007/s00772-014-1431-x

Online publiziert: 13. November 2014

(c) Springer-Verlag Berlin Heidelberg 2014

\title{
H.H. Eckstein
}

Klinik und Poliklinik für Vaskuläre und Endovaskuläre Chirurgie, Klinikum rechts der Isar der Technischen Universität München

\section{Die Zukunft der Zeitschrift Gefässchirurgie}

einem möglichst hohen Niveau informiert werden.

Die Gründungsherausgeber der Gefässchirurgie Hans-Martin Becker und Peter Maurer aus München, Karl Rendl aus Salzburg und Jon Largiader aus Luzern hatten Jens Allenberg auserwählt, dieses gemeinsame Projekt der Deutschen (DGG), Österreichischen (ÖGG) und Schweizerischen (SGG) Gesellschaften für Gefäßchirurgie voranzutreiben. Alle drei Fachgesellschaften benötigten ein neues Publikationsorgan, da die von Peter Maurer seit 1979 herausgegebene Zeitschrift Angio vom Demeter Verlag 1994 aus wirtschaftlichen Gründen eingestellt worden war. Glücklicherweise wurde mit dem Springer Verlag dann rasch ein sehr erfahrener und leistungsfähiger Verlag gefunden.

Die damaligen Gründe für eine eigene Fachzeitschrift gelten heute nahezu unverändert:

- Wissenschaftliche Fachgesellschaften benötigen ein eigenes Publikationsund Mitteilungsorgan, um in regelmäßiger Kommunikation mit ihren Mitgliedern die eigene Identität und fachliche Ausrichtung entwickeln und verstetigen zu können.

- Alle deutschsprachigen vaskulären Forscher und vor allem auch der wissenschaftliche Nachwuchs aus der Gefäßchirurgie sollten unbedingt die Möglichkeit erhalten, auch im deutschsprachigen Raum in einem „peer-review journal“ zu publizieren.

- Die Mitglieder aller drei Fachgesellschaften müssen über neueste Studien und Forschungsfragen fortlaufend auf
- Den Mitgliedern aller drei Fachgesellschaften müssen hochwertige Weiterbildungs- und CME-Artikel zur Verfügung gestellt werden.

Sehr schnell gelang es, unter der Schriftleitung von J.R. Allenberg (1996-2000), S. von Sommogyi (2000-2004) und E.S. Debus (2004-2012) die Gefässchirurgie als Zeitschrift für vaskuläre und endovaskuläre Chirurgie (seit 2007 Zeitschrift für Vaskuläre Medizin) im deutschsprachigen Raum zu etablieren. Die große Mehrzahl aller Gefäßchirurg(inn)en lesen die Gefässchirurgie regelmäßig und beziehen hierbei wichtige Informationen für ihre tägliche Arbeit. Aufgrund der steigenden Mitgliederzahlen in allen drei Fachgesellschaften nahm auch die Auflage von anfangs ca. 1.000 auf zuletzt ca. 3.000 Exemplare zu.

Meilensteine der Gefässchirurgie sind die Erhöhung der Heftzahl von initial 4 auf 6 (2005) bzw. 8 Hefte seit 2009, CMEArtikel seit 2005, Vierfarbigkeit seit 2005 und die Einführung eines Editorial Managers im Jahr 2009. Letzteres hat den internen Review-Prozess deutlich erleichtert. Das jederzeit konstruktive Engagement des Springer Verlags führte im Jahr 2010 zur Einführung des Julius-SpringerPreises für Gefäßmedizin, der jährlich für herausragende translationale Forschungsarbeiten verliehen wird.

Etwas weniger erfolgreich ist leider die Wahrnehmung und Akzeptanz der Gefässchirurgie in der nationalen und internationalen „Gefäßszene“ außerhalb unse- rer Fachgesellschaften. Nachdem es 2009 erstmals gelungen war, beim ISI (Institute for Scientific Information) eine Listung und einen Impact Factor (IF) zu erzielen, wurde dieser vor wenigen Monaten ohne Angabe von Gründen wieder gestrichen. Vermutlich waren die vergleichsweise niedrige Anzahl an Zitationen der Gefässchirurgie in anderen Journalen und die zu niedrige Anzahl an Originalarbeiten ausschlaggebend für diese Entscheidung.

Dies ist natürlich ein herber Rückschlag, zumal wir für den Impact Factor 2013 einen Anstieg errechnet hatten. Man muss nun leider davon ausgehen, dass es für viele Autoren zukünftig weniger interessant wird, in der Gefässchirurgie Forschungsergebnisse oder auch gute Review-Artikel zu publizieren - eine heikle Situation, da hierdurch das Niveau der Gefässchirurgie gefährdet wird.

Hinter den Kulissen haben bereits intensive Diskussionen begonnen, wie es nun mit „unserer“ Gefässchirurgie weitergehen soll. Verschiedene alternative Szenarien sind denkbar:

1. Rückzug auf reine Weiterbildungsinhalte und Verzicht auf künftige Bemühungen einer erneuten Listung bei ISI inkl. Verzicht auf einen offiziellen Impact Factor.

2. Oder: Einrichtung eines zweiten stark wissenschaftlich orientierten OnlineJournals, als Publikationsorgan für unseren akademisch interessierten Nachwuchs.

3. Oder: Schaffung eines sog. HybridJournals mit einer deutschsprachigen gedruckten Ausgabe (wie bisher) und einem Online-Supplement, in wel- 
chem ausgewählte Artikel aus Gefässchirurgie zusätzlich in Englisch und Open Access publiziert werden und damit in PubMed Central im Volltext kostenfrei zugänglich sind. Arbeitstitel dieses Hybrid-Journals wäre $G e$ fässchirurgie International.

In ganz ähnlicher Form hat z. B. das Deutsche Ärzteblatt (DÄB) und das Journal der Deutschen Dermatologischen Gesellschaft (JDDG) den Spagat zwischen anspruchsvoller Weiterbildung für alle und internationaler Sichtbarkeit hervorragend bewältigt. Der Impact Factor dieser Journale steigt übrigens jedes Jahr und liegt für 2013 bei 3,6 (DÄB) bzw. 1,8 (JDDG).

Nach langer und intensiver Diskussion hat sich das Editorial Board der Gefäss chirurgie in seiner Sitzung am 26.09.2014 $\mathrm{zu}>90 \%$ für die Option 3 ausgesprochen. Während Option 1 als prinzipiell nicht zukunftsfähig erachtet wurde, hat Option 2 den gravierenden Nachteil, dass die in einem separaten Online-Journal publizierten Arbeiten nicht mehr automatisch den Leser(inne)n und Mitglieder(inne)n unserer drei Fachgesellschaften zugäng lich gemacht werden. Da fortlaufende Informationen über den wissenschaftlichen Fortschritt und akademisch hochwertige Weiterbildungsartikel aber zu den Kernaufgaben der Gefässchirurgie gehören, erscheint die Einrichtung eines HybridJournals aus deutscher Printversion und englischsprachigen Online-Artikeln als beste Wahl.

Entscheidend bei allen Überlegungen ist natürlich die Frage der Finanzierbarkeit. Für online frei zugängliche Artikel (Open Access) im Rahmen von Abonnementzeitschriften verlangen die Verlage sog. APCs (article processing charges), hinzu kommen Übersetzungskosten. In der Regel werden diese Kosten den Autoren bzw. den publizierenden Institutionen auferlegt, ein Weg, der vor dem Hintergrund der akademisch geringen Attraktivität der Gefässchirurgie derzeit nicht gangbar erscheint. Es muss also investiert werden! Aber wer soll das zahlen?

Nun, zunächst einmal müssen alle Beteiligten (Fachgesellschaften, Verlag, Redaktion) sich $\mathrm{zu}$ diesem gemeinsamen Ziel bekennen und dann einen Fahrplan entwickeln, um diese Ziele wirtschaftlich realisieren zu können. Hierzu gehören finanzielles Entgegenkommen und finanzielle Transparenz. Es wird z.B. die Aufgabe der Schriftleitung sein, wirklich nur international zitierfähige Artikel über Open Access und in englischer Sprache zugänglich zu machen und die Übersetzungskosten für diese Artikel auf ein Minimum zu beschränken. Der Springer Verlag wird ebenfalls finanzielle Zugeständnisse machen müssen, um einen gemeinsamen zukünftigen Erfolg überhaupt zu ermöglichen und - last, but not least werden die drei Fachgesellschaften finanzielle Anstrengungen unternehmen müssen, um die zukünftigen Kosten übernehmen zu können.

In diesem Zusammenhang muss die Entwicklung der Kosten bzw. des Preises betrachtet werden, die von DGG, SGG und ÖGG pro Mitglied für den Bezug der Gefässchirurgie aufzubringen sind. In den ersten Jahren musste investiert werden, um die Gefässchirurgie überhaupt zum Laufen zu bringen. In den folgenden Jahren sind diese Kosten dann u. a. aufgrund steigender Mitgliederzahlen stetig gefallen und machen heute nur noch einen Bruchteil der Investitionen aus der Anfangszeit aus. Diese einerseits erfreuliche Entwicklung bedeutet aber auch, dass bei dem aktuellen Heftpreis von wenigen Euro keinerlei Spielräume für die skizzierte Weiterentwicklung unserer Zeitschrift bestehen.

Aus unserer Sicht ist es daher zwingend notwendig, zukünftig wieder einen höheren Anteil aus den Mitgliedsbeiträgen für die Gefässchirurgie zu verwenden. Möglicherweise ist am Ende der Diskussion - zumindest für die DGG - auch eine moderate Erhöhung der Mitgliedsbeiträge notwendig, vielleicht sollte uns ein gutes Journal dies aber auch wert sein. Nur am Rande: Die Deutsche Dermatologische Gesellschaft verwendet einen vielfach höheren Betrag, um ihr auch international mittlerweile sehr respektiertes Hybrid-Journal „JDDG“ zu unterstützen. In diesem Zusammenhang sei erwähnt, dass eine spontane Umfrage auf der Mitgliederversammlung am 24.07.2014 in Hamburg eine überwältigende Mehrheit für eine Beitragserhöhung zur Finanzierung einer Online-Version unserer Zeitschrift ergeben hat.
Liebe Leserinnen und Leser der Gefässchirurgie,

alle genannten Szenarien sind natürlich Überlegungen, die in den letzten Monaten nach ausführlichen Recherchen bei aktuell auf dem Markt befindlichen Online-Anbietern, mit dem Springer Verlag, den Herausgebern und den Vorständen der Fachgesellschaften intensiv diskutiert worden sind. Während die SGG sich diesen Plänen sofort anschließen konnte, gibt es bei der DGG und der ÖGG Sorgen wegen der finanziellen Implikationen, vereinzelt auch Meinungen, die den Status quo akzeptieren möchten (bzw. sich für Option 1 aussprechen).

Aus diesem Grund wollen wir die Leser und Leserinnen der Gefässchirurgie nun gerne direkt befragen. Über den nachfolgenden Link gelangen Sie zu einer kurzen Online-Umfrage. Bitte machen Sie mit, damit wir ein möglichst repräsentatives Meinungsbild erheben können. Alle Angaben bleiben selbstverständlich anonym.

https://www02.surveygrid.com/online. php?p=gech14

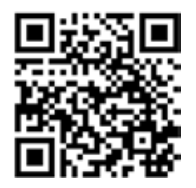

\section{Losungswort: gech14}

Persönlich sind wir davon überzeugt, dass wir für den deutschsprachigen Raum ein national und international wahrnehmbares und respektiertes Journal brauchen. Wir alle erleben täglich den fortwährenden Umbruch in unserem Fach, der klinisch und akademisch nur dann nachhaltig erfolgreich sein wird, wenn wir über die eigenen Grenzen schauen und uns auch international einbringen. Die deutschsprachigen Länder verfügen über sehr gute klinische Daten und relevante translationale Forschungsprojekte, die es lohnt, einem internationalen Leserkreis zur Kenntnis zu bringen. Aus zahlreichen persönlichen Gesprächen weiß ich, dass sich die internationale Gefäßszene sehr viel mehr für Daten und Studien aus dem deutschsprachigen Raum 
interessiert, als wir es uns vielleicht vorstellen können. Außerdem benötigen wissenschaftliche Fachgesellschaften ein in der Fachwelt respektiertes Publikationsorgan, um den eingangs gestellten Aufgaben (Identität, Möglichkeit zur Publikation in einem deutschsprachigen Journal, Informationsplattform für Mitglieder der Fachgesellschaften) erfolgreich nachkommen zu können. Somit sehen wir für $G e$ fässchirurgie International trotz aller aktuellen Widrigkeiten ein erhebliches $\mathrm{Zu}$ kunftspotenzial, mit einer realistischen Chance, in nicht so ferner Zukunft wieder einen Impact Factor zu erhalten und dann endlich auch in MEDLINE gelistet zu werden. Packen wir es an!

Mit den besten kollegialen Grüßen Ihr

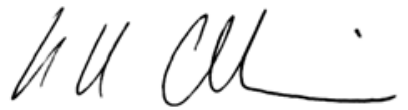

H.H. Eckstein

\section{Korrespondenzadresse}

Prof. Dr. H.H. Eckstein

Klinik und Poliklinik für Vaskuläre und Endovaskuläre Chirurgie, Klinikum rechts der Isar der Technischen Universität München Ismaninger Str. 22, 81675 München hheckstein@web.de

Interessenkonflikt. H.H. Eckstein gibt an, dass kein Interessenkonflikt besteht.

\section{Visualisierung von medizinischen Untersuchungs- ergebnissen}

Wissenschaftler der Jacobs University Bremen wollen die Formen der Visualisierung von medizinischen Untersuchungsergebnissen verbessern. Diese Visualisierungen können Ungenauigkeiten enthalten, die den Arzt zu einer falschen Diagnose oder Behandlung veranlassen, die mit genaueren Darstellungen nicht notwendig wären.

Besonderer Forschungsschwerpunkt ist das Erkennen und die Diagnose von arteriellen Gefäßverengungen im Halsbereich. Ob eine Verengung besteht, können Ärzte nur anhand von Darstellungen beurteilen, die aus MRTAufnahmen generiert werden. Fehlerquellen können durch Bewegung des Patienten bei den Aufnahmen oder durch eine fehlerhafte Erzeugung der dreidimensionalen Daten entstehen. Mit ihrer Arbeit wollen die Wissenschaftler feststellen, wie groß die Unsicherheiten in den generierten Bildern sind, und möchten die Mediziner für dies Unsicherheiten sensibilisieren. Ebenfalls möchten sie analysieren, welche visuellen Kennzeichnungen sich für die zweifelhaften Regionen eignen.

Quelle: Jacobs University Bremen, www.jacobs-university.de

\section{Krebsmedikament gegen Krampfadern}

Ein Krebsmedikament kann die Verengung der Blutgefäße durch chronisch hohem Blutdruck und auch Entstehung von Krampfadern verhindern. Diese Entdeckung machte eine Wissenschaftlerin des Universitätsklinikum Heidelberg.

Dr. Larissa Pfisterer entdeckte, dass sobald der Druck auf die Gefäßwände dauerhaft ansteigt, ein Mechanismus um Myokardin außer Kraft gesetzt wird. Myokardin ermöglicht, dass Blutgefäße Blutdruckschwankungen durch eine kurzfristige Anpassung ihres Durchmessers ausgleichen. Unter chronisch hohem Druck wird es derart verändert, dass es die Funktion verliert und abgebaut wird. In Folge wächst die Muskelschicht in den Gefäßwänden überproportional an, so dass die Gefäße sich dauerhaft verengen.

Die neuen Erkenntnisse betreffen nicht nur Arterien, die sich erhöhtem Blutdruck anpassen müssen, sondern auch überlastete Venen z.B. in den Beinen. Frau Dr. Pfisterer zeigte, dass vergleichbare Mechanismen an der Bildung von Krampfadern beteiligt sind. Derzeit untersucht sie mögliche Therapieansätze - mit ersten Erfolgen: Bei Mäusen verhindert schon eine sehr geringe Dosis des Krebsmedikaments Bortezomib die schädliche Kettenreaktion in den Gefäßwänden und damit die Veränderungen der Venen.

Für die in ihrer Doktorarbeit erlangten Erkenntnisse wurde Frau Dr. Pfisterer mit dem Friedrich Reutner-Preis ausgezeichnet.

Literatur: Pfisterer L, Meyer R, Feldner A et al (2014) Bortezomib protects from varicose-like venous remodeling. FASEB J. 28:3518-27.

Quelle: Universitätsklinikum Heidelberg. www.klinikum.uni-heidelberg.de 